\title{
A new method for the determination of the coefficient of thermal expansion of solid materials
}

\author{
by $\underline{\text { R. Montanini* }}$, F. Freni*
}

*Dept. of Industrial Chemistry and Materials Engineering, University of Messina, Viale F. Stagno D'Alcontres 3198166 Messina, Italy, rmontanini@unime.it, fabfreni@unime.it

\begin{abstract}
A new experimental method (IIC, Infrared Image Correlation) for quick assessment of the coefficients of thermal expansion of solid materials is presented. The proposed method is based on performing a digital image correlation between thermal images acquired at different temperatures by means of an infrared camera. It allows the coefficient of thermal expansion of both isotropic and anisotropic bulk materials to be determined by measuring simultaneously the fractional increase in length and the actual thermal field over a small region of interest in which a dual-emissivity stochastic speckle pattern has been created. First results reported in this paper prove the effectiveness of the proposed method that can be applied either to carry out reference measurements in laboratory or to evaluate thermal stresses and strains on structural components in-field.
\end{abstract}

\section{Introduction}

In its simplest form, the average (i.e., defined over a temperature range) coefficient of linear thermal expansion (CTE) of any material can be defined as the fractional increase in length (linear dimension) per unit rise in temperature. The most general definition is the mean or average coefficient of thermal expansion as given by ASTM [1-2]:

$$
\alpha_{m, 1}=\frac{\left(L_{2}-L_{1}\right) / L_{0}}{T_{2}-T_{1}}=\frac{1}{L_{0}} \cdot \frac{\Delta L}{\Delta T}
$$

Here $\alpha_{m, 1}$ is related to the slope of the chord between two points on the curve of length against temperature, and so represent the expansion over the particular temperature range from $T_{1}$ to $T_{2}$, with initial length $L_{0}$ defined at $a$ reference temperature $T_{0}$. Often the lower limit of the specified temperature range is also used as the reference temperature, leading to a modification of the former equation to the following [3]:

$$
\alpha_{m, 2}=\frac{\left(L_{2}-L_{1}\right) / L_{1}}{T_{2}-T_{1}}=\frac{1}{L_{1}} \cdot \frac{\Delta L}{\Delta T}
$$

CTE is a temperature-dependent property and will, for most metals and alloys, gradually increase with temperature. From Eqs. (1) and (2), it can be noted that the CTE refers to a temperature range and not to a single temperature.

According to the previous formulae, determination of the average coefficient of thermal expansion requires the measurement of two physical quantities, displacement and temperature, which can be accomplished by means of different experimental methods. Standard methods include mechanical dilatometry [1], laser interferometry [2] and high magnification optical imaging [3]. The increasing use of computer-based thermo-mechanical models as well as specific requirements concerning anisotropic materials has determined in the last few years a greater need for quantitative data, promoting the development of new full-field optical measurement techniques, such as those based on Electronic Speckle Pattern Interferometry (ESPI) [4-5], phase-shifting interferometry [6] and digital image correlation [7]. All these techniques differs from each other only for the way the material expansion is measured with, since the reference temperature is always determined by monitoring the air temperature inside the heating device used to exert thermal loading, rather than measuring it directly on the sample surface.

In this paper a new non-contact method which allows some of the limitations associated with the existing techniques to be overcome is introduced. The proposed method (IIC) relies on recording two (or more) infrared images at two (or more) different temperatures of a random dual-emissivity speckle pattern created over a small region of the target surface and by post-processing the resulting infrared images by means of a digital image correlation (DIC) algorithm in order to provide the full-field in-plane displacement map of the thermally loaded sample surface. To prove the feasibility and effectiveness of the proposed method, the CTE in $x$ and $y$ directions of a nearly isotropic aluminium alloy specimen and a anisotropic unidirectional glass fiber reinforced composite specimen were measured and compared with values obtained by means of a reference commercial dilatometer. 


\section{Materials and methods}

\subsection{Infrared image correlation (IIC) technique}

Digital image correlation is an optical experimental technique originally developed by a group of researchers at the University of South Carolina in the 1980s [8-9] and since then applied in numerous applications in the field of experimental mechanics [10]. It directly provides full-field in-plane displacement fields of the test planar specimen surface by comparing digital images recorded in the un-deformed (or reference) and deformed states, respectively. The basic approach relies on random speckle patterns sprayed onto the test surface which deforms together with it and acts as a carrier of deformation information.

The working principle of the proposed measurement technique, which relies on digital image correlation, can be illustrated synthetically as follows (figure $1 \mathrm{a})$. A subset of $(2 \mathrm{M}+1) \times(2 \mathrm{M}+1)$ pixels centred at point $\left(\mathrm{x}_{0}, \mathrm{y}_{0}\right)$ from the reference infrared image at temperature $T_{0}$ is first chosen and used to determine its corresponding location in the deformed image (at temperature $\mathrm{T}_{\mathrm{N}}$ ). A sum-square difference (SSD) correlation algorithm is then applied to evaluate the similarity between the reference subset and the target subset. The matching procedure is carried out numerically through searching the peak position of the correlation coefficients distribution. Once the maximum of the correlation function has been detected, the target subset in the two images can be determined, allowing the displacement field to be calculated (figure $1 b)$. In practical implementation, a region of interest (ROI) in the reference image is specified first and divided into evenly spaced virtual grids. In-plane displacements were computed at each point of the virtual grids to obtain the full-field deformation, while the corresponding reference temperatures were provided by the infrared camera. Finally, the average CTEs in $x$ and $y$ directions were calculated using Eq. (2) based on the average thermal strains extracted from the thermal displacement fields.

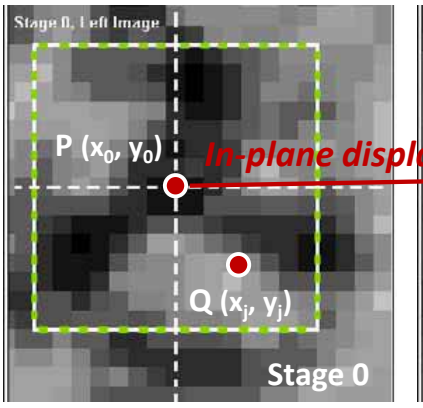

Reference image $\left(T_{0}\right)$

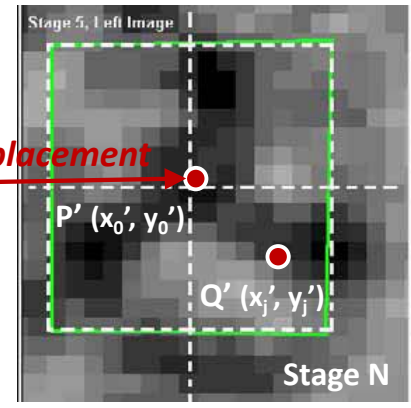

Deformed image $\left(T_{N}\right)$
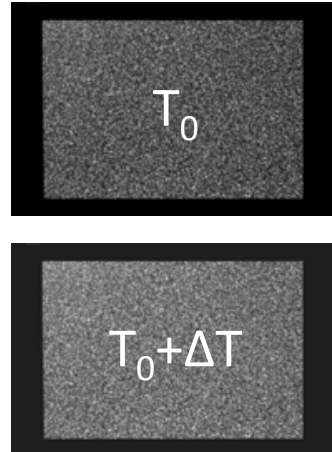
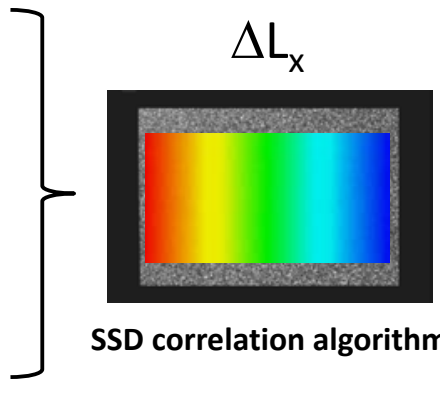

SSD correlation algorithm

b)

Fig. 1. a) Schematic illustration of a square subset before (Stage 0) and after (Stage $N$ ) thermal loading, the differences of positions of the reference subset center and target subset center yield in-plane displacement components; b) Reconstructed false colours surface displacement field (x component)

\subsection{Realization of the random speckle pattern}

As infrared images in the MWIR range are used, in order to make "visible" the speckle pattern the physics of thermal radiation must be exploited. In particular, an IR signature could be obtained by using materials with different emissivity. In addition, several requirements must be met to make the infrared image correlation technique effective:

- the surface must exhibit a suitable stochastic speckle pattern in order to clearly allocate the pixels in the thermal images. Thus, a pixel area in the reference image can be allocated to the corresponding pixel area in the target image;

- the surface pattern must be able to follow the thermal deformation of the specimen and must not break early neither cause a reinforcing effect on the test sample (i.e., its thickness and stiffness should be negligible with respect to those of the test specimen);

- the materials used to create the speckle pattern should have a low thermal expansion coefficient (i.e., they must not expand when exposed to relatively large $\Delta T$ );

- the materials used to create the speckle pattern must display good contrast in the MWIR spectral band because otherwise the matching procedure (correlation) does not work;

- the average emissivity of the surface pattern should be as high as possible to prevent spurious reflections that can affect the accuracy of temperature measurements. Reflections also cause a bad contrast in the thermal images preventing matching computation in the areas of reflection;

- on one hand, the size of the surface characteristics should be small enough to allow a fine raster of calculation subsets during evaluation. On the other hand, the pattern should be large enough to be completely resolved by the infrared camera; 
- the specimen surface must be flat and oriented orthogonally to the axis of the camera. The proposed IIC method can also be used for measuring the CTE of non-planar surfaces (i.e., 3D geometries) by employing two image acquisition systems (stereoscopic setup) instead than one, allowing all the three components of the displacement vector to be determined by tracking the motion of each image point in the field of view.

Several trials were made in order to find the best suitable solution for creating the speckle pattern. Good results were achieved with fine-grained gypsum powders applied over a uniform layer of opaque black paint sprayed onto the target surface in order to create a high emissivity $\left(\varepsilon_{\text {paint }}=0.96\right)$ background. Gypsum has in fact a very low emissivity coefficient $\left(\varepsilon_{\text {gypsum }} \cong 0.1\right)$ in the $3-5 \mu \mathrm{m}$ spectral range, producing a high infrared contrast with respect to the black paint used as background. In addition, gypsum is a non-metallic material and does not expand with temperature. Figure 2 shows some examples of dual-emissivity speckle patterns created on the surface of the two test specimens used to carry out the experiments.

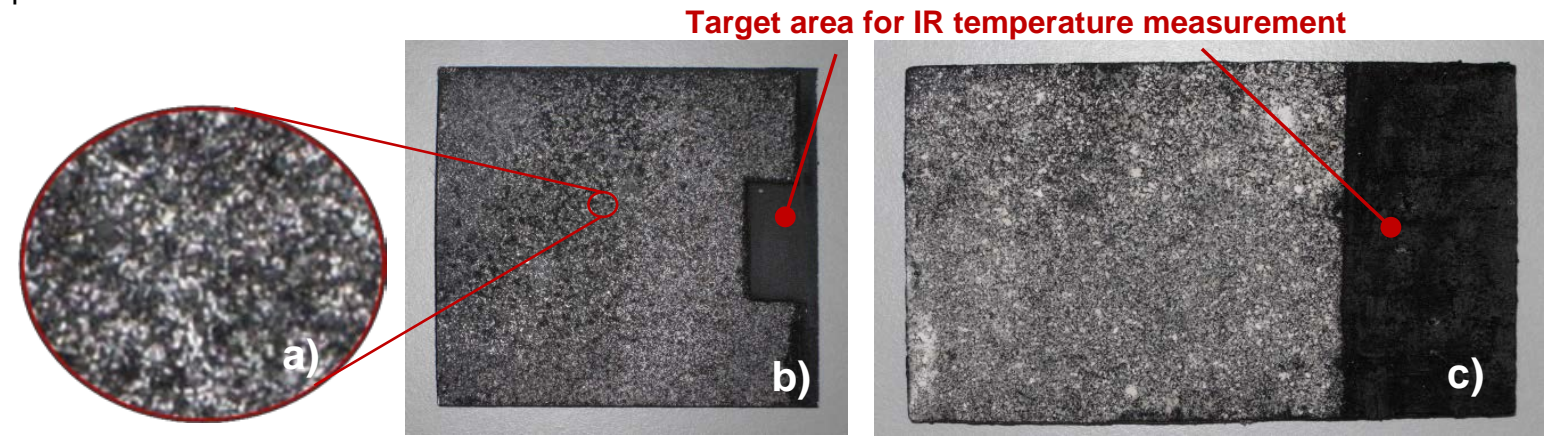

Fig. 2. Dual-emissivity stochastic speckle patterns obtained by depositing fine-grained gypsum powder over a uniform layer of high emissivity opaque black paint: a) close-up view; b) Al alloy sample; c) anisotropic GFRP sample.

\subsection{Heating device}

A dedicated heating device (figure 3) was designed to carry out the experimental tests. It basically consists of a thermally insulated chamber equipped with an electronic temperature controller. Heat insulation was achieved by using 6 $\mathrm{mm}$ thick ceramic sheets made from calcium silicate, which were carefully cut to the desired dimension. The heating source consists of two electrical resistances mounted laterally with respect to the specimen, which was retained horizontally on two small cylindrical supports without restraints. To prevent direct radiation, the heating sources have been "obscured" using thermally opaque screens. The heating device was instrumented with a Pt100 thermometer used to control the air temperature inside it. Two further surface type $\mathrm{K}$ thermocouples were mounted on the top and on the bottom sides of the specimen for monitoring the bulk temperature and checking for potential thermal gradients across its thickness. An optical access was realized on the top of the chamber using a zinc selenide (ZnSe) window (figure 4a). Although the heating chamber was designed by taking into account the possibility of making a partial vacuum inside it, first experiments were carried out at atmospheric pressure. The heating device can operate up to $200{ }^{\circ} \mathrm{C}$.

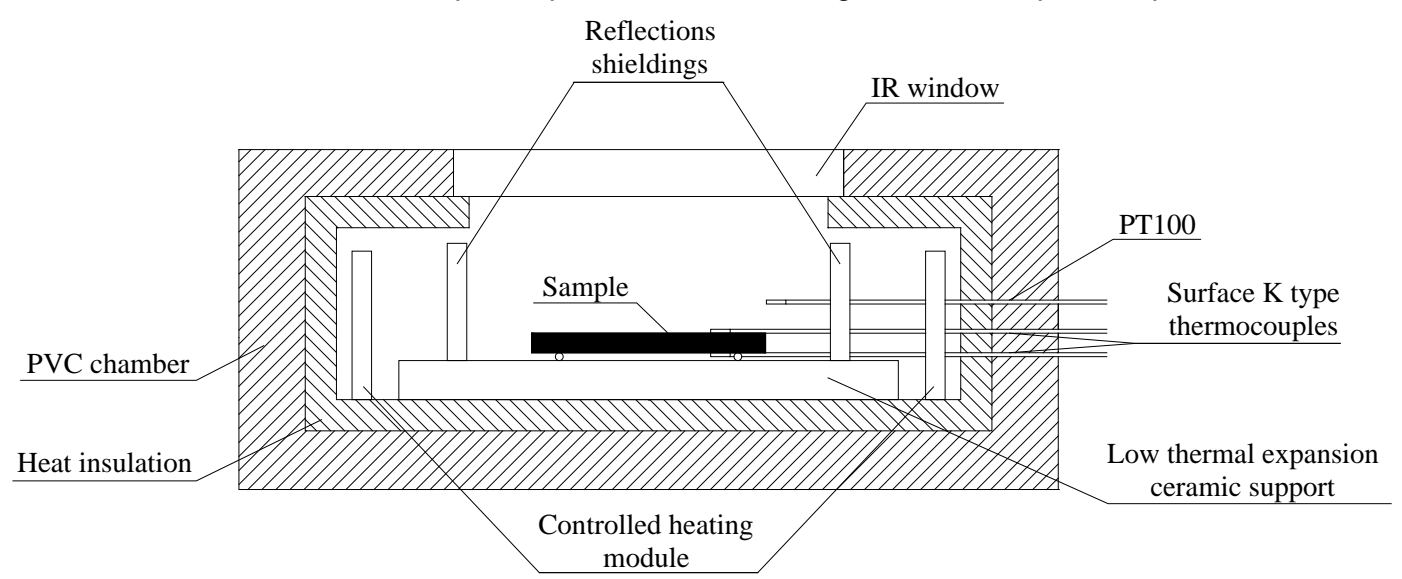

Fig. 3. Schematic drawing of the heating device used to carry out the experimental tests.

Measurement of the surface specimen temperature (figure 4b) was accomplished by means of an InSb cooled $640 \times 512$ pixels infrared camera (Cedip Titanium), operating in the MWIR range and equipped with a $50 \mathrm{~mm}$ lens. The IR camera was carefully aligned with respect to the heating device, that was placed on an optical table. Preliminary tests were performed to calibrate the temperature values measured across the ZnSe window, taking into account its actual IR transmittivity. All the experiments were conducted in a semi-anechoic chamber specifically developed by the authors. 

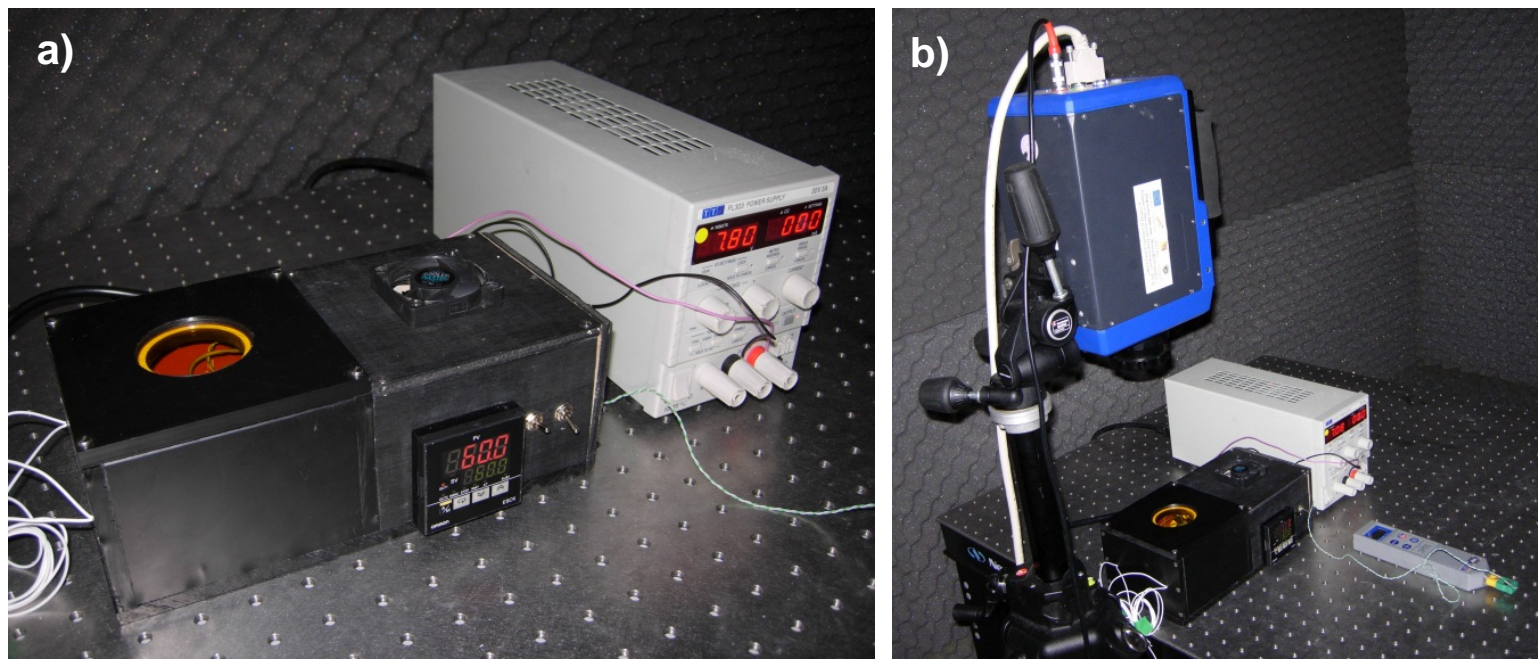

Fig. 4. a) Heating device used to conduct the experimental tests; b) Measurement set-up.

\subsection{Specimens}

Two different specimens were analysed. The first one is made of aluminum alloy, the second one of unidirectional glass fiber reinforced composite. It is expected that, while the aluminium alloy specimen should exhibit a near isotropic behaviour when subjected to a thermal load, the composite specimen should not instead, manifesting a markedly anisotropic thermal strain field. Both specimens have similar dimensions, roughly $60 \times 40 \times 2 \mathrm{~mm}(\mathrm{~W} \times \mathrm{L} \times \mathrm{H})$. The speckle pattern was created on the whole specimens surface except than on a small rectangular that was only black painted. This part of the sample, which is within the field of view of the IR camera, was used as target area for the measurement of $\Delta T$.

\subsection{Experimental procedure}

Experimental tests were carried out in steady state conditions using multiple thermal steps from $30^{\circ} \mathrm{C}$ to $60^{\circ} \mathrm{C}$, with $5^{\circ} \mathrm{C}$ increments. For each temperature level, a suitable stabilization time (variable from 10 to 20 min) was waited for allowing thermal equilibrium of the specimen to be reached. Ten infrared images (8 bit gray levels) were recorded at each stabilized temperature level and then stored for further post-processing. The first temperature step was used as reference for the computation of $\Delta T$ and $\Delta L$. Each sequence of seven images (one for each temperature step) was then processed numerically in order to retrieve the actual values of $\Delta T$ and $\Delta L$. Mean temperatures were obtained for each step by averaging the temperature values measured by the IR camera within the blackened small rectangular area made on the specimen surface. Relative displacements $\Delta L$ in $x$ and $y$ directions were calculated, after image calibration, at a mesh grid of $225(15 \times 15)$ points, with subset size of $61 \times 61$ pixels and 20 pixel overlapping. Relative displacements computed by means of infrared image correlation are influenced by unavoidable small rigid body translations [11] that occur during the thermal expansion of the test specimen, which was placed into the heating device without restraint. This effect must be corrected in order to get pure thermal expansion of the test specimen. This was done by subtracting the displacements of the center point of the calculation area to each image of the recorded sequence. Conversely, rigid body rotations of the test specimen, which result in contours lines of $x$ and $y$ displacement fields that are no longer parallel with axis $y$ and $x$, do not affect strain components [7] and were not compensated for in image processing. The displacement measurement accuracy, that can be controlled by adjusting the subset size, was estimated as 0.02 pixels in our experiments (or $1.48 \mu \mathrm{m}$, as the actual magnification of the imaging system was about $74 \mu \mathrm{m} /$ pixel). Average strain components along $x$ and $y$ directions were finally computed by numerically differentiating the displacement fields. Image correlation was carried out using the software Aramis by GOM mbH (Braunschweig, Germany).

To assess the effectiveness of the IIC method, the obtained CTE values were compared with those measured on the same specimens by means of a commercial pushrod dilatometer (NETZSCH DIL 402 C).

\section{Preliminary results}

Pure (after rigid body motion compensation) thermal displacement fields of Al alloy along $x$ and $y$ directions are reported in figure 5 for $\Delta T=30^{\circ} \mathrm{C}$ (at $T_{2}=60^{\circ} \mathrm{C}$ ). The maximum displacement caused by thermal expansion is about 21.3 $\mu \mathrm{m}$ in the $x$ direction and $20.1 \mu \mathrm{m}$ in the $y$ one. As the small difference observed between the two values is within the displacement measurement uncertainty, it can be concluded that Al alloy exhibits, as expected, a nearly isotropic behavior when subjected to thermal loading. The resultant strain maps are shown in figure 6. 

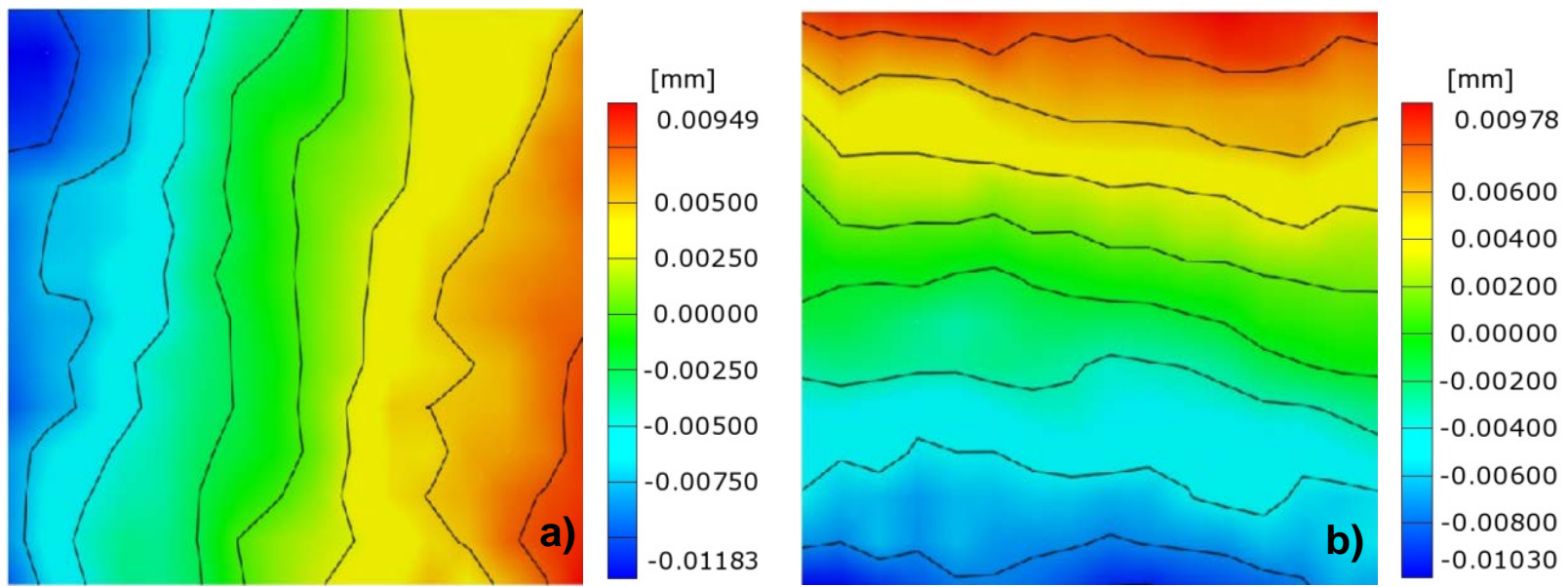

Fig. 5. Thermal displacement fields of Al alloy sample after rigid body motion compensation: a) $x$ displacement field at $T_{2}=60^{\circ} \mathrm{C}\left(\Delta T=30^{\circ} \mathrm{C}\right) ;$ b) y displacement field at $T_{2}=60^{\circ} \mathrm{C}\left(\Delta T=30^{\circ} \mathrm{C}\right)$.
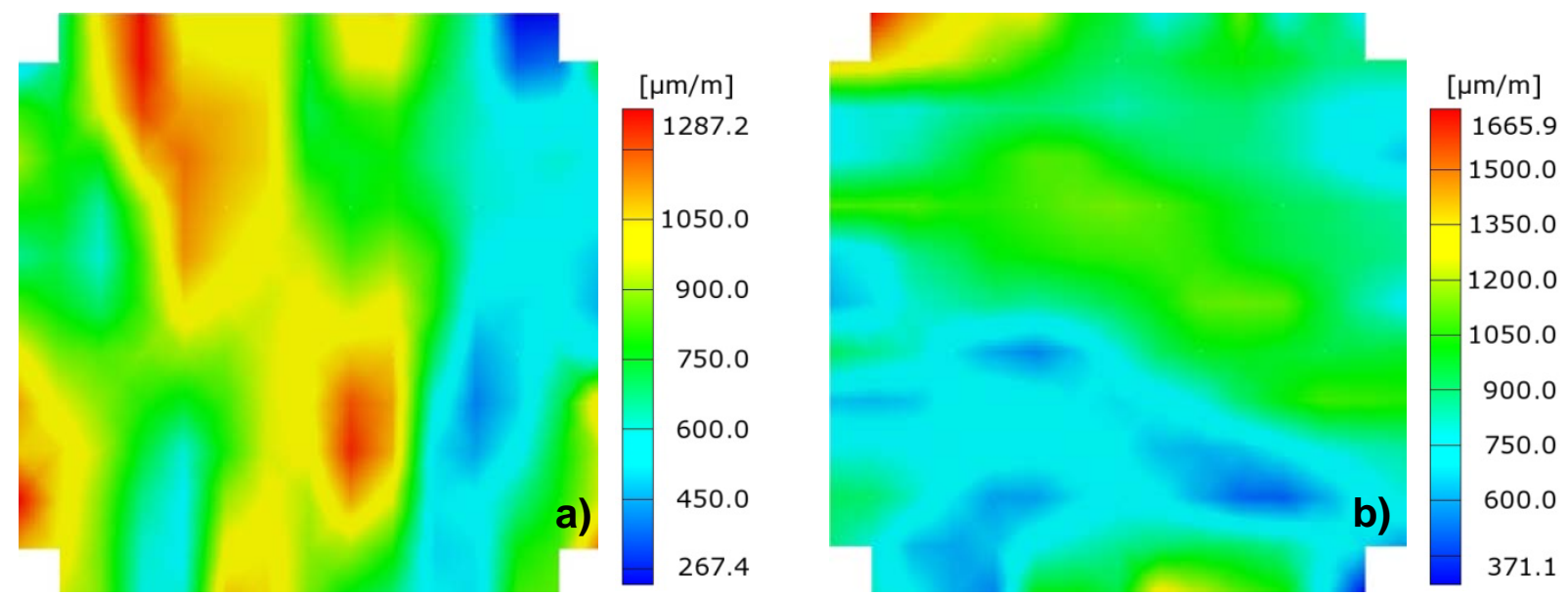

Fig. 6. Thermal strain fields of Al alloy sample after rigid body motion compensation: a) $x$ strain field at $T_{2}=60^{\circ} \mathrm{C}$ $\left(\Delta T=30^{\circ} \mathrm{C}\right) ;$ b) y strain field at $T_{2}=60^{\circ} \mathrm{C}\left(\Delta T=30^{\circ} \mathrm{C}\right)$.

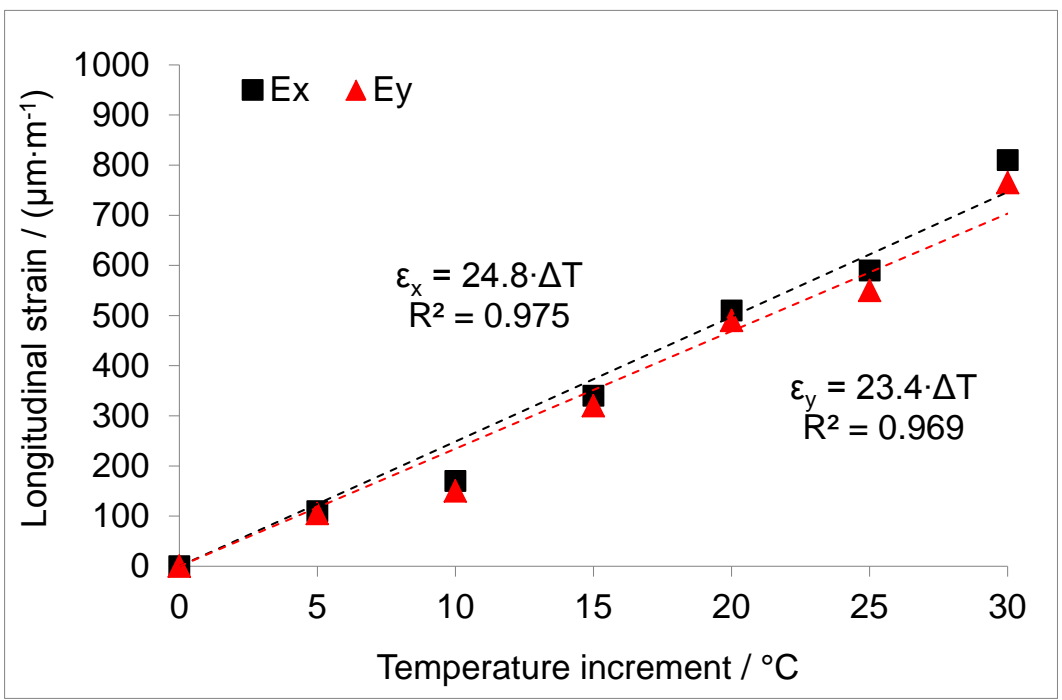

Fig. 7. Temperature-strain curves of Al alloy measured in the $30-60^{\circ} \mathrm{C}$ temperature range. 

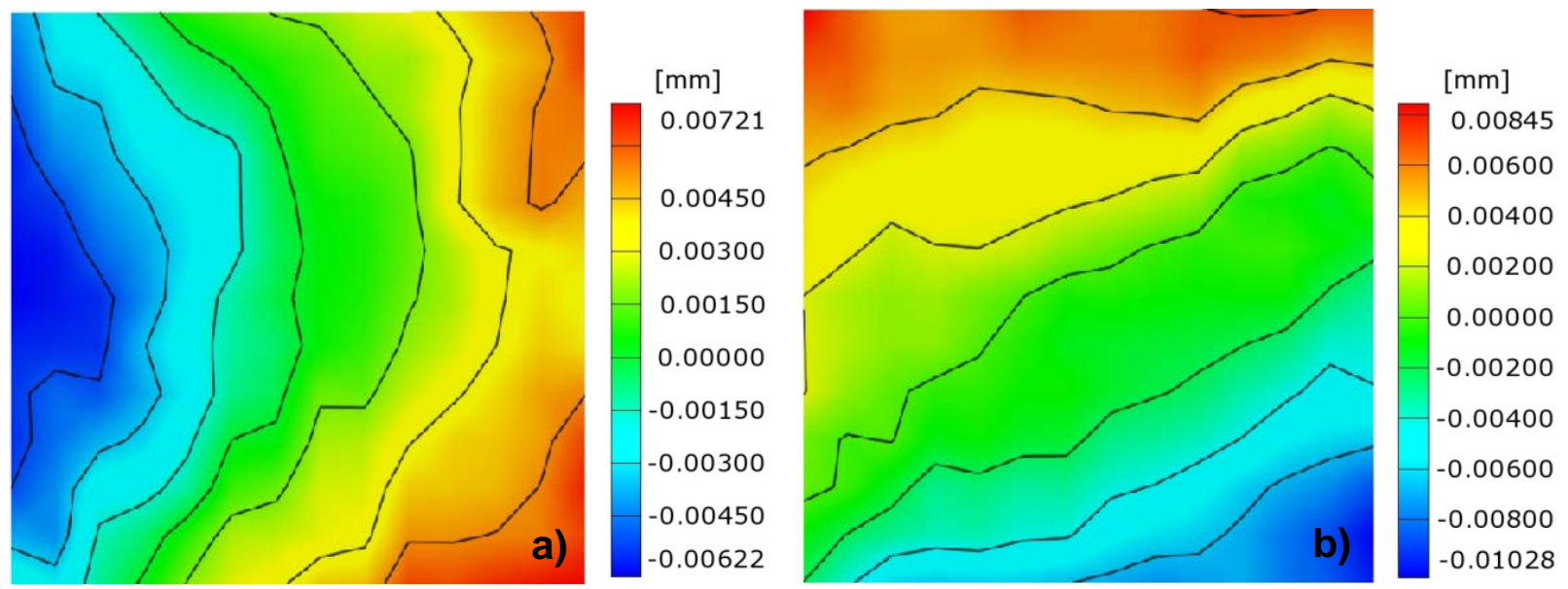

Fig. 8. Thermal displacement fields of unidirectional GFRP sample after rigid body motion compensation: a) $x$ displacement field at $T_{2}=60^{\circ} \mathrm{C}\left(\Delta T=30^{\circ} \mathrm{C}\right) ;$ b) y displacement field at $T_{2}=60^{\circ} \mathrm{C}\left(\Delta T=30^{\circ} \mathrm{C}\right)$.
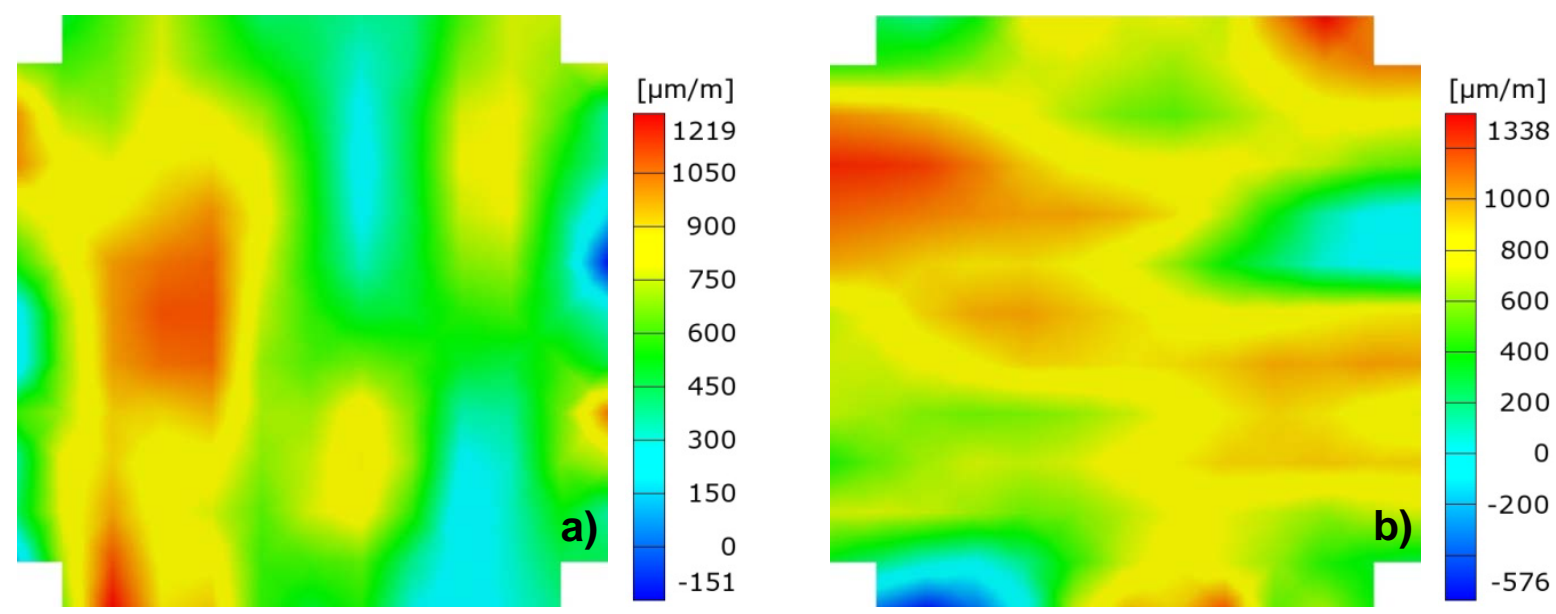

Fig. 9. Thermal strain fields of unidirectional GFRP sample after rigid body motion compensation: a) $x$ strain field at $T_{2}=60^{\circ} \mathrm{C}\left(\Delta T=30^{\circ} \mathrm{C}\right) ;$ b) y strain field at $T_{2}=60^{\circ} \mathrm{C}\left(\Delta T=30^{\circ} \mathrm{C}\right)$.

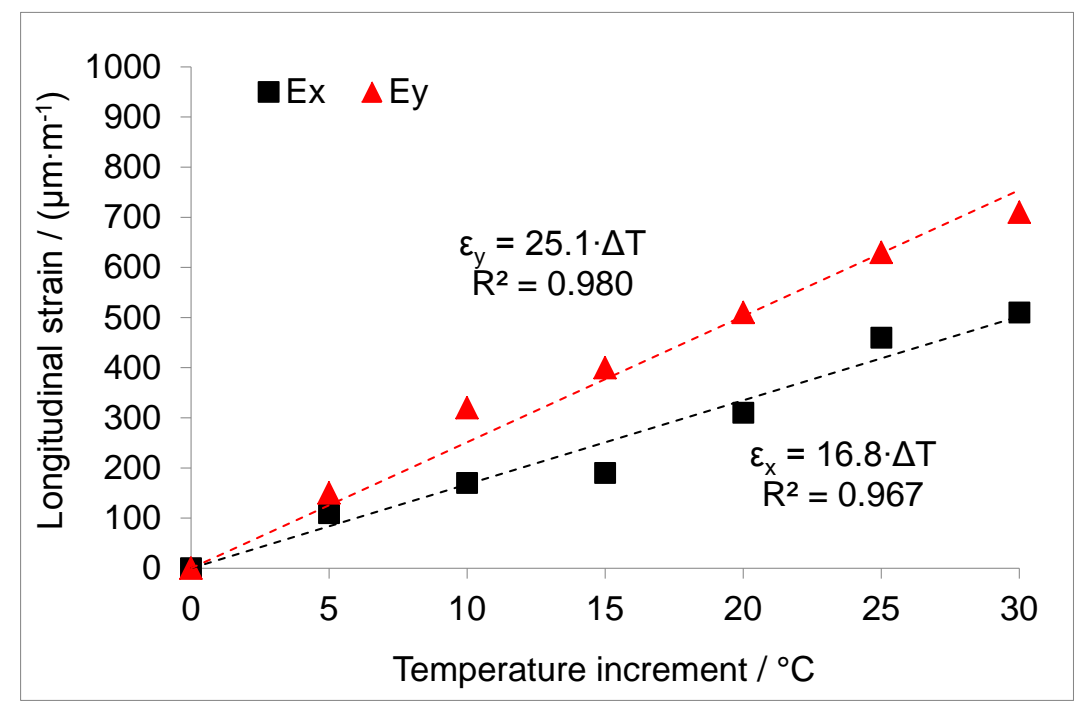

Fig. 10. Temperature-strain curves of unidirectional GFRP measured in the $30-60^{\circ} \mathrm{C}$ temperature range. 
Table 1. Prospect of the obtained results and comparison with pushrod dilatometry (DIL).

\begin{tabular}{|c|c|c|c|c|c|c|c|c|}
\hline & & & \multicolumn{3}{|c|}{ Al alloy } & \multicolumn{3}{|c|}{ Unidirectional $(x)$ GFRP } \\
\hline $\mathrm{T}_{1}\left({ }^{\circ} \mathrm{C}\right)$ & $\mathrm{T}_{2}\left({ }^{\circ} \mathrm{C}\right)$ & $\Delta \mathrm{T}\left({ }^{\circ} \mathrm{C}\right)$ & \multicolumn{2}{|c|}{$\alpha_{\| I C}\left(\mu \mathrm{m} \mathrm{m}^{-1}{ }^{\circ} \mathrm{C}^{-1}\right)$} & $\alpha_{\mathrm{DIL}}\left(\mu \mathrm{m} \mathrm{m}{ }^{-1}{ }^{\circ} \mathrm{C}^{-1}\right)$ & $\alpha_{\| C}$ & $\left.{ }^{-1}{ }^{\circ} \mathrm{C}^{-1}\right)$ & $\alpha_{\mathrm{DIL}}\left(\mu \mathrm{m} \mathrm{m}^{-1}{ }^{\circ} \mathrm{C}^{-1}\right)$ \\
\hline \multirow{2}{*}{30} & \multirow{2}{*}{60} & \multirow{2}{*}{30} & $x$ & 23.4 & \multirow{2}{*}{23.7} & $x$ & 16.8 & 16.1 \\
\hline & & & $y$ & 24.8 & & $y$ & 25.1 & 25.8 \\
\hline
\end{tabular}

Local spots (noise) caused by numerical differentiation of the displacement fields are observable in the strain maps, especially nearby the boundary of the computation area. Actually, the accuracy of strain field computation strongly depends on the spatial resolution of the IR imaging system, as will be discussed with more details in the next section. Average thermal strain values $\left( \pm\right.$ std dev.) of $(810 \pm 220) \mu \mathrm{m} / \mathrm{m}$ and $(765 \pm 200) \mu \mathrm{m} / \mathrm{m}$ were obtained at $60^{\circ} \mathrm{C}(\Delta T=$ $30^{\circ} \mathrm{C}$ ) along $x$ - and $y$-direction, respectively. To quantitatively determine the CTEs of Al alloy sample, the experimental data were fitted by a straight line (figure 7 ).

Pure (after rigid body motion compensation) thermal displacement fields of GFRP along $x$ and $y$ directions are reported in figure 8 for $\Delta T=30^{\circ} \mathrm{C}\left(\right.$ at $T_{2}=60^{\circ} \mathrm{C}$ ). The maximum displacement caused by thermal expansion is about 13.4 $\mu \mathrm{m}$ in the $x$ direction and $18.7 \mu \mathrm{m}$ in the $y$ one. Thus, it can be highlighted that the GFRP sample exhibits a markedly anisotropic behavior when subjected to thermal loading. The resultant strain maps are shown in figure 9 . As already noted for the Al alloy sample, strain images are affected by noteworthy local noise arising from the numerical differentiation process. Average thermal strain values $( \pm$ std dev.) of $(510 \pm 250) \mu \mathrm{m} / \mathrm{m}$ and $(710 \pm 320) \mu \mathrm{m} / \mathrm{m}$ were obtained at $60^{\circ} \mathrm{C}\left(\Delta T=30^{\circ} \mathrm{C}\right)$ along $x$ - and $y$-direction, respectively. To quantitatively determine the CTES of GFRP sample, the experimental data were fitted by a straight line (figure 10).

The obtained results are summarized in Table 1. A good agreement (within the actual experimental uncertainty) can be observed between CTE values measured by means of the proposed method and those determined using conventional pushrod dilatometry.

\section{Discussion}

According to Eq. (2), the accuracy of CTE evaluation by the IIC method can be estimated, as a first approximation, by propagating the uncertainty contributes of $\Delta \varepsilon$ and $\Delta T$ :

$$
u(\alpha)=\sqrt{\sum_{i=1}^{n}\left(\frac{\partial \alpha}{\partial x_{i}}\right)^{2} \cdot u^{2}\left(x_{i}\right)}=\sqrt{\left(\frac{\partial \alpha}{\partial(\Delta \varepsilon)}\right)^{2} \cdot u^{2}(\Delta \varepsilon)+\left(\frac{\partial \alpha}{\partial(\Delta T)}\right)^{2} \cdot u^{2}(\Delta T)}
$$

where $u(\Delta \varepsilon)$ and $u(\Delta T)$ are standard uncertainties associated to $\Delta \varepsilon$ and $\Delta T$, respectively. Typically, the accuracy of the displacement estimation using a SSD correlation algorithm is \pm 0.02 pixels [10]. Therefore, the accuracy of thermal strain computation can be estimated as $|0.02+0.02| / L$ by forward difference, where $L$ is the initial length at a reference temperature. As in our experiments $L$ is about 355 pixels, then, assuming a rectangular distribution between the lower and upper bounds, $u(\Delta \varepsilon)$ can be estimated to be around $65 \mathrm{ppm}$. The accuracy of temperature difference measurement by means of the infrared camera can be estimated as twice its NETD value, hence $u(\Delta T)$ is about $0.03^{\circ} \mathrm{C}$ if a symmetric rectangular probability distribution is subjectively assumed. By applying Eq. (3), it follows that the expected combined uncertainty of CTE will be roughly $\pm 2.2 \mathrm{ppm}^{\circ} \mathrm{C}^{-1}$ (note that this value might be reduced drastically by increasing $\Delta T$ ).

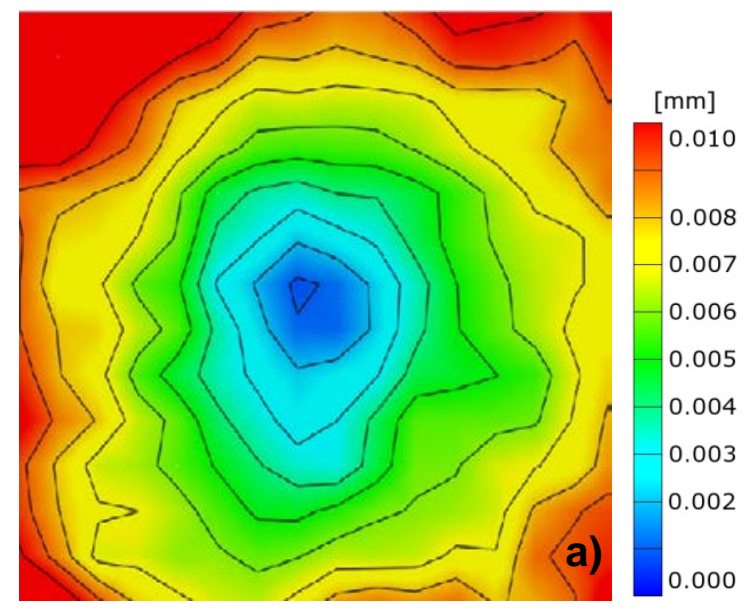

Fig. 11. Surface vector displacement maps measured at $T_{2}=60^{\circ} \mathrm{C}\left(\Delta T=30^{\circ} \mathrm{C}\right)$ : a) Al alloy sample; b)

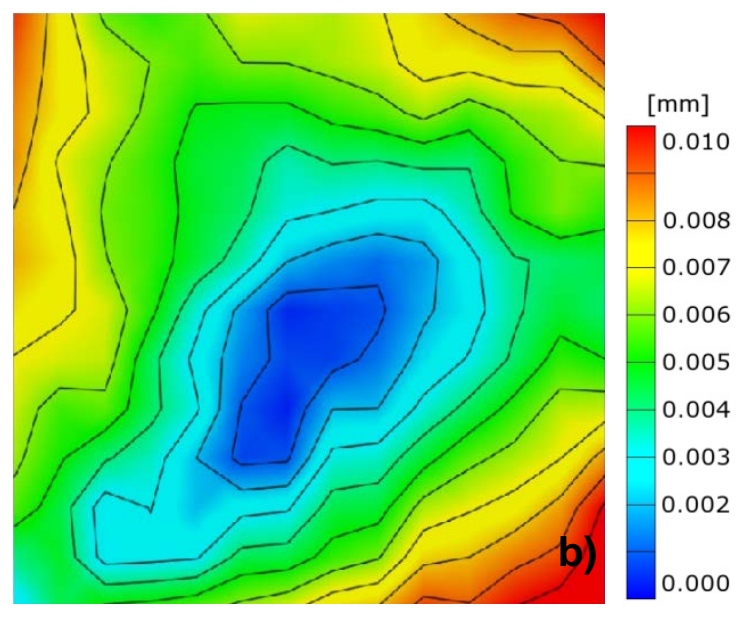
unidirectional GFRP sample. 
The main contribution to $u(\alpha)$ derives from $u(\Delta \varepsilon)$. Therefore, in order to reduce the uncertainty of CTE, $L$ should be made as large as possible. Because of the limited spatial resolution of conventional IR cameras, this will either require using large specimens or close-up lens to exploit the entire field of view of the imaging system.

One main benefit of the proposed approach is the possibility of determining average CTE values in multiple directions with a single test. This is an important feature when dealing with materials characterized by a markedly anisotropic behavior, as it is the case with most composite structures. Figure 11 illustrates the surface vector displacement fields of $\mathrm{Al}$ alloy and GFRP specimens obtained for a temperature difference of $30^{\circ} \mathrm{C}$. The different response of the two materials to a thermal load is clearly revealed by observing the reconstructed false-colours maps and the degree of anisotropy can be easily assessed (table 1).

Opposite to other experimental methods employed for assessment of materials CTE, IIC makes use of the actual temperature difference measured directly on the specimen surface, rather than relying on the nominal temperature regulated by the heating controlling system, thus eliminating systematic errors caused by a non-uniform temperature distribution on the testing material.

In addition, since IIC is a full-field technique, it might also be used to assess the true coefficient of linear expansion (usually referred to as thermal expansivity) of a material subjected to a non-uniform unidirectional thermal flow, by measuring the derivatives $d L / d T$ at specific sections of the sample surface. Future work is envisaged to demonstrate the practical feasibility of this challenging task. Currently, the on-going research is focused on the optimization of the measurement set-up. This will encompass, in addition to enhancement of the spatial resolution of the imaging system by using a close-up lens, several aspects which include optimization of the dual-emissivity speckle pattern, assessment of the influence of image brightening with temperature and alleviation of noise-related problems associated to the computation of the thermal strain field.

\section{Conclusions}

In this paper a new method for quick assessment of the coefficients of thermal expansion of bulk materials has been introduced. The method, which has been called Infrared Image Correlation (IIC), is based on performing a digital image correlation between thermal images acquired at different temperatures by means of an infrared camera. First results reported in this paper prove the effectiveness of the proposed approach that can be applied either to carry out reference measurements in laboratory or to evaluate thermal stresses and strains on structural components in-field

\section{REFERENCES}

[1] American Society for Testing and Materials 2011 ASTM E 228-11.

[2] American Society for Testing and Materials 2010 ASTM E 289-04

[3] James J. D., Spittle J. A., Brown S.G.R., Evans R.W., "A review of measurement techniques for the thermal expansion coefficient of metals and alloys at elevated temperatures", Meas. Sci. Technol., vol. 12, pp. R1-R15, 2001

[4] Kim S., Kim J. H., Lee J. K., Jarng S. S., "Measurement of thermal expansion coefficients by electronic speckle pattern interferometry at high temperature", J. Mater. Sci. Lett., vol. 16, pp. 1753-56, 1997.

[5] Dudescu C., Naumann J., Stockmann M., Nebel S., "Characterisation of thermal expansion coefficient of anisotropic materials by electronic speckle pattern interferometry", Strain, vol. 42 (3), pp.197-205, 2006.

[6] Lee C.C., Tien C.L., Sheu W.S., Jaing C.C., "An apparatus for the measurement of internal stress and thermal expansion coefficient of metal oxide films", Rev. Sci. Instrum., vol. 72 (4), pp.2128-2133, 2001.

[7] Bing P., Hui-min X., Tao H., Asundi A., "Measurement of coefficient of thermal expansion of films using digital image correlation method", Polymer Testing, vol. 28, pp.75-83, 2009.

[8] Peters W., Ranson W., "Digital imaging techniques in experimental stress analysis", Opt. Eng.; vol. 21 (3), pp. 427-431, 1982.

[9] Sutton M., Wolters W., Peters W., McNeill S., "Determination of displacements using an improved digital correlation method", Image Vision Comput., vol. 1, pp. 133-139, 1983.

[10] Pan B., Qian K., Xie H., Asundi A., "Two-dimensional digital image correlation for in-plane displacement and strain measurement: a review", Meas. Sci. Technol., vol. 20, pp. 1-17, 2009.

[11] Haddadi H., Belhabib S., "Use of rigid-body motion for the investigation and estimation of the measurement errors related to digital image correlation technique", Opt. Laser Eng., vol. 46, pp. 185-196, 2008. 